\title{
Targeted tumor imaging of anti-CD20-polymeric nanoparticles developed for the diagnosis of B-cell malignancies
}

This article was published in the following Dove Press journal:

International Journal of Nanomedicine

22 June 2015

Number of times this article has been viewed

\section{Sara Capolla' \\ Chiara Garrovo \\ Sonia Zorzet' \\ Andrea Lorenzon ${ }^{3}$ \\ Enrico Rampazzo ${ }^{4}$ \\ Ruben Spretz ${ }^{5}$ \\ Gabriele Pozzato 6 \\ Luis Núñez \\ Claudio Tripodo 8 \\ Paolo Macor ${ }^{1,9}$ \\ Stefania Biffi ${ }^{2}$}

'Department of Life Sciences, University of Trieste, ${ }^{2}$ Institute for Maternal and Child Health IRCCS "Burlo Garofolo", Trieste,

${ }^{3}$ Animal Care Unit, Cluster in Biomedicine (CBM scrl), Trieste, Italy; ${ }^{4}$ Department of Chemistry "G. Ciamician”, University of Bologna, Bologna, Italy; ${ }^{5}$ LNK Chemsolutions LLC, Lincoln, NE, USA; ${ }^{6}$ Department of Medical, Surgery and Health Sciences, University of Trieste, Trieste, Italy; ${ }^{7}$ Bio-Target, Inc., University of Chicago, Chicago, IL, USA;

${ }^{8}$ Department of Human Pathology, University of Palermo, Palermo, Italy;

${ }^{9}$ Callerio Foundation Onlus, Institutes of Biological Researches, Trieste, Italy

Correspondence: Stefania Biffi Institute for Maternal and Child Health - IRCCS "Burlo Garofolo", via dell'Istria, 65/I, 34/37 Trieste, Italy Tel +390403785478

Fax +39040 3785210

Email stefania.biffi@burlo.trieste.it

\begin{abstract}
The expectations of nanoparticle (NP)-based targeted drug delivery systems in cancer, when compared with convectional therapeutic methods, are greater efficacy and reduced drug side effects due to specific cellular-level interactions. However, there are conflicting literature reports on enhanced tumor accumulation of targeted NPs, which is essential for translating their applications as improved drug-delivery systems and contrast agents in cancer imaging. In this study, we characterized biodegradable NPs conjugated with an anti-CD20 antibody for in vivo imaging and drug delivery onto tumor cells. NPs' binding specificity mediated by anti-CD20 antibody was evaluated on MEC1 cells and chronic lymphocytic leukemia patients' cells. The whole-body distribution of untargeted NPs and anti-CD20 NPs were compared by time-domain optical imaging in a localized human/mouse model of B-cell malignancy. These studies provided evidence that NPs' functionalization by an anti-CD20 antibody improves tumor pharmacokinetic profiles in vivo after systemic administration and increases in vivo imaging of tumor mass compared to non-targeted NPs. Together, drug delivery and imaging probe represents a promising theranostics tool for targeting B-cell malignancies.
\end{abstract}

Keywords: active targeting, optical imaging, tumor accumulation

\section{Introduction}

Nanomedicine for cancer therapy is advantageous over conventional medicine ${ }^{1}$ because it has the potential to enable the preferential delivery of drugs to tumors owing to the enhanced permeability and retention (EPR) effect, and the delivery of more than one therapeutic agent for combination therapy. ${ }^{2}$ Moreover, the engineering of a targeting ligand onto the nanoparticles' (NPs') surface can result in prolonged and localized drug delivery, which should translate into greater efficacy and reduced drug side effects. ${ }^{3-5}$

Cancer treatment stands to benefit from targeted drug delivery, as tumor cells express many molecules on their surface that distinguish them from normal cells. ${ }^{6}$ Although there are expectations of improved efficacy of targeted NPs derived from specific cellular-level interactions, there are conflicting literature reports on tumor accumulation of targeted NPs: previous reports suggest that a tumor-targeting ligand does not increase NP accumulation in tumors, but the targeting antibody may increase tumor cell internalization. ${ }^{3,7}$ The results of these studies suggest that the tumor localization of NPs is independent of the ligand and primarily relies on the EPR effect. ${ }^{8}$ These reports are in contrast, however, to others, which suggest that the attachment of tumor-targeting antibody on NPs enhances total accumulation in solid tumor. ${ }^{9-11}$ One difference might be the presence or absence of polyethylene glycol (PEG) in the complex and its impact on the EPR effect. ${ }^{12}$ In fact, the increased circulation time 
and tumor retention time of the PEGylated complexes could result in the enhanced tumor accumulation of both targeted and untargeted complexes, thereby masking any effect of the ligand in the process. EPR is a highly variable phenomenon, characterized by large inter- and intraindividual differences. In light of this, a significant number of tumor-targeting studies have focused on the evaluation of parameters affecting the EPR effect, ${ }^{13,14}$ and additional studies are clearly needed to understand the influence of NPs' modification on tumor localization and uptake. . $^{3,7-11}$

Currently, several nanotechnology-enabled diagnostic and therapeutic agents for cancer are in clinical trials. ${ }^{15}$ Indeed, multifunctionality is the key feature of NP-based agents. Targeting ligands, imaging labels, therapeutic drugs, and other functionalities can all be integrated to allow for targeted molecular imaging and cancer molecular therapy. ${ }^{16}$

Recently, we focused on identifying "next-generation" treatments for B-cell disorders ${ }^{17,18}$ that currently take advantage of dose-intensive chemotherapy regimens and immunotherapy via use of monoclonal antibodies. We developed a novel therapeutic approach based on hydroxychloroquine- and chlorambucil-loaded anti-CD20 NPs, which selectively inhibits tumor growth in mice bearing B-cell lymphoma xenograft.

In this work, the whole-body distribution of Cy5.5untargeted NPs and Cy5.5-anti-CD20 NPs was determined by time-domain (TD) optical imaging (OI) in a localized animal model of B-cell malignancy, thus providing insight for the NP behavior in vivo as imaging probe. Our studies provide evidence that NPs' functionalization by an anti-CD20 ligand and imaging agent improves tumor pharmacokinetic profiles in vivo after systemic administration and represent a promising imaging tool for the diagnosis of B-cell malignancies.

\section{Material and methods}

\section{Synthesis and characterization of NPs}

Chemicals used for NP preparation were reagent grade or better. Some of the purchased chemicals included polyethylene glycol (PEG Nektar, San Carlos, CA, USA) Hydroxychloroquine sulfate (HCQ, ACROS, Gel, Belgium) and Chlorambucil (CLB, Sigma Aldrich, St Louis, MO). NPs based on carboxylic acid-terminated biodegradable polymers (PLA-b-PEG-COOH and PCL-COOH) were produced with an expected average diameter of $250 \mathrm{~nm}$. NPs were fabricated under class 100 clean-room conditions using a proprietary electrohydrodynamic technology (Bio-Target Inc., Chigaco, IL, USA; LNK Chemsolutions LLC, Lincoln, Nebraska, USA). Briefly, for all NP formulations, organic solutions containing all the necessary components were processed using this technology resulting in a dry collection of the specified
NPs. The collected material was then harvested in an aqueous buffer solution to obtain a stable suspension. ${ }^{18}$ NPs were suspended in $0.1 \mathrm{M}$ sodium carbonate buffer $(\mathrm{pH}=9.3)$ and stained with FluoroLink ${ }^{\mathrm{TM}}$ Cy5.5 Monofunctional Dye (GE Healthcare Bio-Sciences AB, Uppsala, Sweden) for 30 minutes. To eliminate the excess of dye, NPs were dialyzed. The amount of Cy5.5 associated with the NP surface was quantified by spectrophotometric methods. Molar extinction coefficients of $250,000 \mathrm{M}^{-1} \mathrm{~cm}^{-1}$ at $678 \mathrm{~nm}$ for the Cy5.5 dye were used.

NPs' hydrodynamic diameter $\left(\mathrm{d}_{\mathrm{H}}\right)$ distributions and $\zeta$-potential values were determined using a Malvern Nano $\mathrm{ZS}$ instrument as previously described. ${ }^{19}$

\section{$\mathrm{MECI}$ and chronic lymphocytic leukemia cell microscopy}

MEC1 cell line (kindly provided by Professor Josee Golay) was cultured in RPMI-1640 medium (Sigma-Aldrich Co., St Louis, MO, USA) supplemented with 10\% fetal bovine serum (Thermo Fisher Scientific, Waltham, MA, USA). Heparinized peripheral blood samples were obtained after informed consent from eight B-chronic lymphocytic leukemia (CLL)-untreated patients characterized as such in Table S1, and the mononuclear cell fractions were isolated by centrifugation on Ficoll-Hypaque (GE Healthcare) density gradients. ${ }^{20}$ For fluorescence staining, MEC1 cells and CLL-patients' cells were suspended in serum-free RPMI-1640 medium and stained with Vybrant ${ }^{\mathrm{TM}}$ DiI cell-labeling solution (GE Healthcare) for 20 minutes. The excess of dye was eliminated by centrifugation. To label nuclei, MEC1 cells were stained with DAPI (SigmaAldrich Co.) for 5 minutes. Finally, the samples were cytocentrifuged on positive-charged glasses (Bio-Optica, Milan, Italy) and analyzed using a Leica DM2000 fluorescence microscope (Leica Microsystems, Wetzlar, Germany). In addition, electron microscopy samples were treated as previously described by preparing ultrathin sections with an Ultratome III (Pharmacia LKB, Uppsala, Sweden) which were analyzed by transmission electron microscopy (TEM) (EM208; Philips, Eindhoven, the Netherlands). ${ }^{21}$ Micrographs were acquired with a Morada Camera (Olympus Corporation, Tokyo, Japan).

\section{Flow cytometric analysis}

The immunophenotypical characterization of MEC1 and CLL-affected patients' cells was made by incubating $5 \times 10^{5}$ cells with anti-human CD45 fluorescein isothiocyanate (FITC) (ImmunoTools, GmbH, Friesoythe, Germany), antihuman CD5 PE (Phycoerythrin) (ImmunoTools), anti-human CD19 PE (ImmunoTools), or anti-CD79a PE (BD, Franklin Lakes, NJ, USA) monoclonal antibodies. To study CD20 
expression, cells were incubated with the anti-CD20 chimeric monoclonal antibody rituximab (Hoffman-La Roche Ltd., Basel, Switzerland) derived from the clinic (University of Trieste, Trieste, Italy) and the anti-human IgG1 FITC (Sigma-Aldrich Co.) antibody. NPs' binding was assayed after incubating MEC1 and CLL-patients' cells with $20 \mu \mathrm{L}$ of NPs for 1 hour at $37^{\circ} \mathrm{C}$. Both experiments were analyzed by FACSCalibur (BD) flow cytometer and data were analyzed by CELLQuest software (BD).

\section{Mice}

Female SCID mice (4-6 weeks old) were purchased from Harlan (San Pietro Al Natisone, Udine, Italy) and maintained under pathogen-free conditions with ad libitum food and water, in accordance with the guide for the care and use of laboratory animals. Xenograft-bearing mice were established through subcutaneous (sc) injection on the left flank of $10^{7}$ MEC1 cells resuspended in $150 \mu \mathrm{L}$ of phosphate-buffered saline. All the experimental procedures were performed in compliance with the guidelines of European and Italian laws and were approved by the institutional animal ethical care committee of the University of Trieste and by the Italian Ministry of Health.

\section{In vivo and ex vivo $\mathrm{Ol}$}

All in vivo data was acquired by using the small-animal TD Optix MX2 preclinical near-infrared(NIR) fluorescence-imager (Advanced Research Technologies, Montreal, Canada), equipped with a pulsed laser diode and a time-correlated single-photon counting detector, as previously described. ${ }^{22,23}$ Briefly, mice were shaved prior to the scanning procedure in order to reduce scattering of the signal from hair. Throughout all imaging sessions, mice were anesthetized with vaporized isoflurane at 1.8\%-2.0\% volume (Biological Instruments, Besozzo VA, Italy). Two-dimensional regions of interest were selected, and laser power, integration time (repetition time of the excitation per raster point), and scan step size were optimized according to the emitted signal. The data were recorded as temporal point-spread functions and the images were reconstructed as fluorescence intensity maps. Prior to injection of the NPs, mice were scanned to obtain background images. For ex vivo OI, the last in vivo whole-body imaging session was followed by euthanasia of animals. The organs were explanted and imaged with the same Optix system. ${ }^{24}$

\section{Tumor tissue analyses}

For each mouse $(n=6)$, tumor size was assessed thrice per week by caliper measurement. Tumor volume was calculated as follows:

$$
\text { Volume }=\mathrm{D} \times \mathrm{d}^{2} \times \pi / 6
$$

where $\mathrm{D}$ and $\mathrm{d}$ are the longer and the shorter diameters, respectively. After ex vivo OI acquisitions, explanted organs and sc tumor masses were fixed in 10\% bufferedformalin solution and embedded in paraffin for histological analysis, or embedded in OCT (optimal cutting temperature) compound embedding medium (Miles, Inc., Diagnostics Division, Elkhart, IN, USA) and snap-frozen at $-80^{\circ} \mathrm{C}$ for fluorescence microscopy. For confocal microscopy analysis, sections of $30 \mu \mathrm{m}$ thickness were cut from frozen organs with a cryostat at $-20^{\circ} \mathrm{C}$ and analyzed using confocal microscope Eclipse te300 (Nikon Corporation, Tokyo, Japan). Immunohistochemical analysis of the tumoral masses were performed with hematoxylin and eosin to evaluate the tissue morphology, and with the avidin-biotin-peroxidase complex to localize CD5, CD20, CD45, and CD79a antigens. ${ }^{25}$ The slides were examined under a Leica DM2000 optical microscope (Leica Microsystems).

\section{Statistical analysis}

Results from at least three independent experiments are reported as the means \pm standard deviation and analyzed for statistical significance by the unpaired Welch two-sample $t$-test. Differences were considered significant when $P$-value was $<0.05$.

\section{Results \\ Synthesis and characterization of NPs}

Two different types of NPs, called untargeted NPs and antiCD20 NPs, were prepared. In detail, untargeted NPs were made only by PLA-b-PEG-COOH and PCL-COOH as polymeric carriers, and anti-CD20 NPs were prepared conjugating the anti-CD20 chimeric antibody rituximab on the surface of untargeted NPs. Morphological characterization by TEM images (Table 1) indicated a core diameter of $110 \pm 40 \mathrm{~nm}$ for untargeted NPs and a core diameter of $90 \pm 30 \mathrm{~nm}$ for

Table I Size distribution and surface potential characterization of NPs

\begin{tabular}{lll}
\hline & $\begin{array}{l}\text { Untargeted } \\
\text { NPs }\end{array}$ & $\begin{array}{l}\text { Anti-CD20 } \\
\text { NPs }\end{array}$ \\
\hline$d_{\text {core }} \pm \mathrm{SD}(\mathrm{nm})$ & $110 \pm 40$ & $90 \pm 30$ \\
Hydrodynamic diameter $^{\mathrm{a}} \pm \mathrm{SD}^{\mathrm{b}}$ & $190 \pm 60$ & $230 \pm 70$ \\
$($ PdI $)(\mathrm{nm})^{\mathrm{c}}$ & $(0.58)$ & $(0.57)$ \\
$\zeta$-potential $\pm \mathrm{SD}(\mathrm{mV})^{\mathrm{c}}$ & $-7.8 \pm 0.9$ & $-6.0 \pm 0.6$ \\
$\zeta_{\text {-potential } \pm \mathrm{SD}(\mathrm{mV})^{\mathrm{d}}}$ & $-13.7 \pm 0.7$ & $-10.5 \pm 0.5$ \\
\hline
\end{tabular}

Notes: ${ }^{a}$ Number mean intensity diameter. ${ }^{b} n=15$. In PBS I $\times, \mathrm{pH} 7.4, \mathrm{BSA} 0.3 \%$. In PBS I×, pH 7.4, BSA 0.3\%, KCl I mM.

Abbreviations: $d_{\text {core }}$, core diameter; NPs, nanoparticles; PBS, phosphate-buffered saline; Pdl, polydispersity index; SD, standard deviation; BSA, bovine serum albumin. 
anti-CD20 NPs. The hydrodynamic diameter measured with dynamic light scattering (Table 1) was $190 \pm 60 \mathrm{~nm}$ for untargeted NPs, and 230 $\pm 70 \mathrm{~nm}$ for anti-CD20 NPs, with a polydispersity index of 0.58 and 0.57 , respectively, indicating a slight aggregation among particles.

The prepared NPs were suspended in phosphate-buffered saline $\mathrm{pH} 7.4$ to give a concentration of $1.66 \mathrm{mg} / \mathrm{mL}$ polymers, $8.8 \mu \mathrm{g} / \mathrm{mL}$ anti-CD20 antibody, and $2.8 \mathrm{nmol} / \mathrm{mL}$ Cy5.5.

\section{Characterization of MECI and NPs' binding}

MEC1 cells were characterized by evaluating the expression of surface antigens, such as CD5, CD19, CD20, CD45,
A

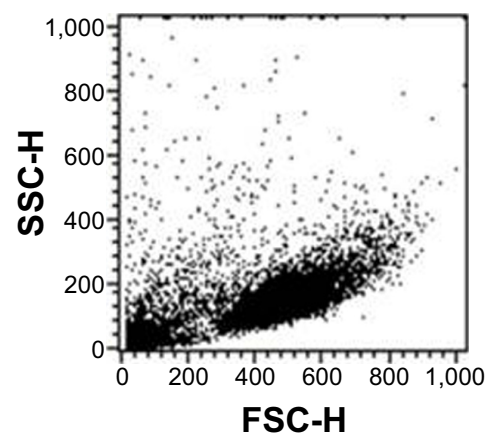

CD20

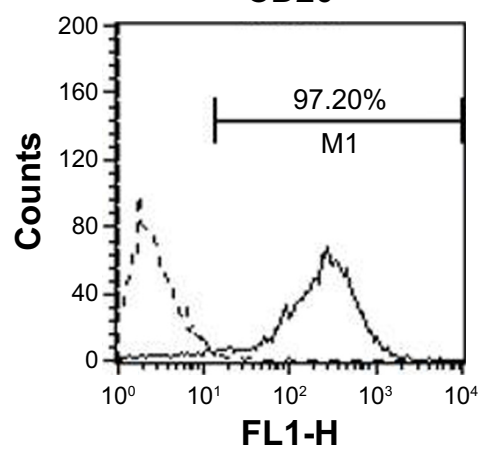

B

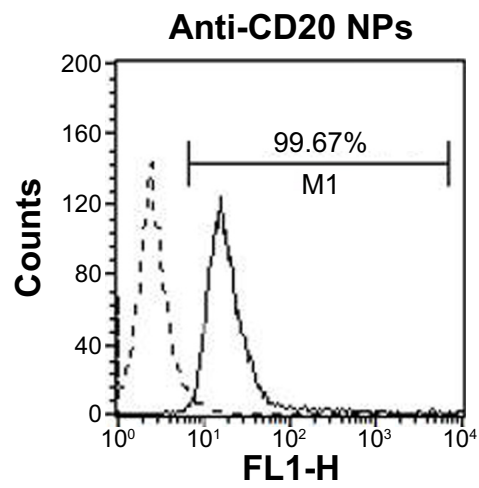

CD5

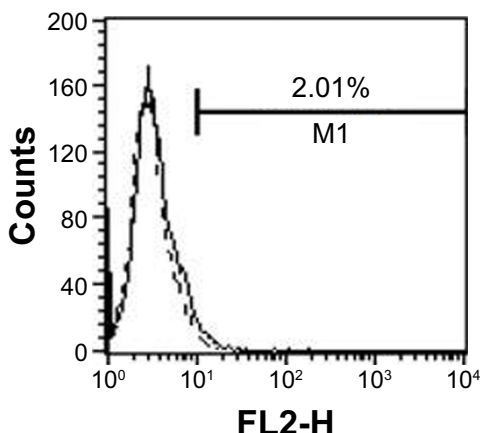

CD45
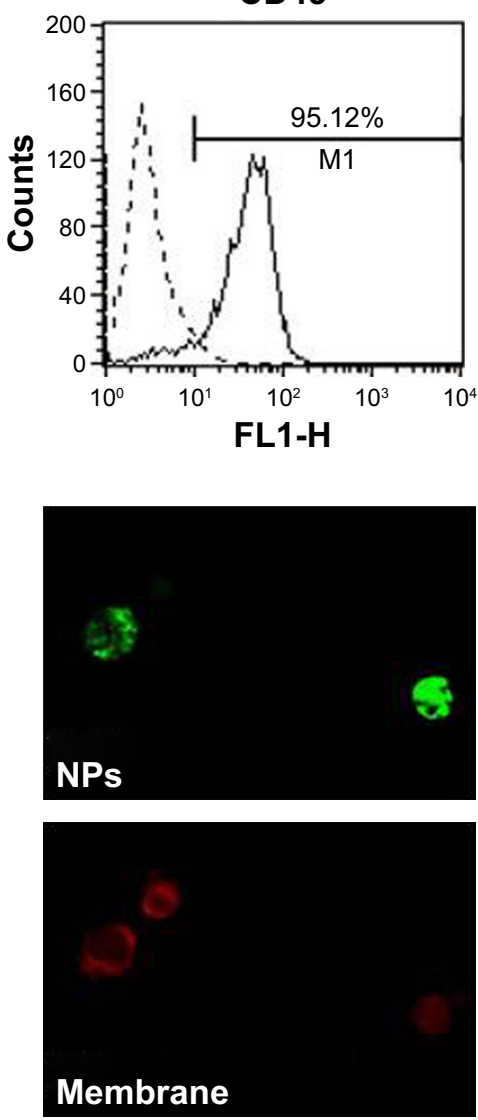

CD19

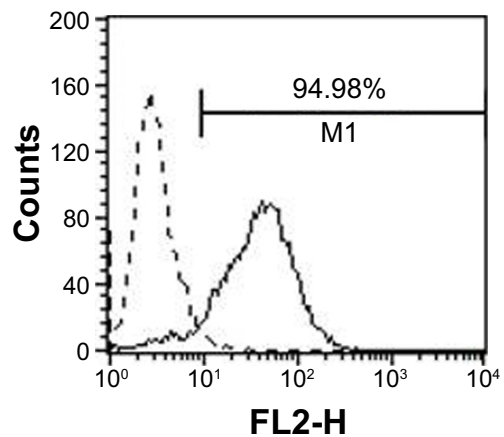

CD79a
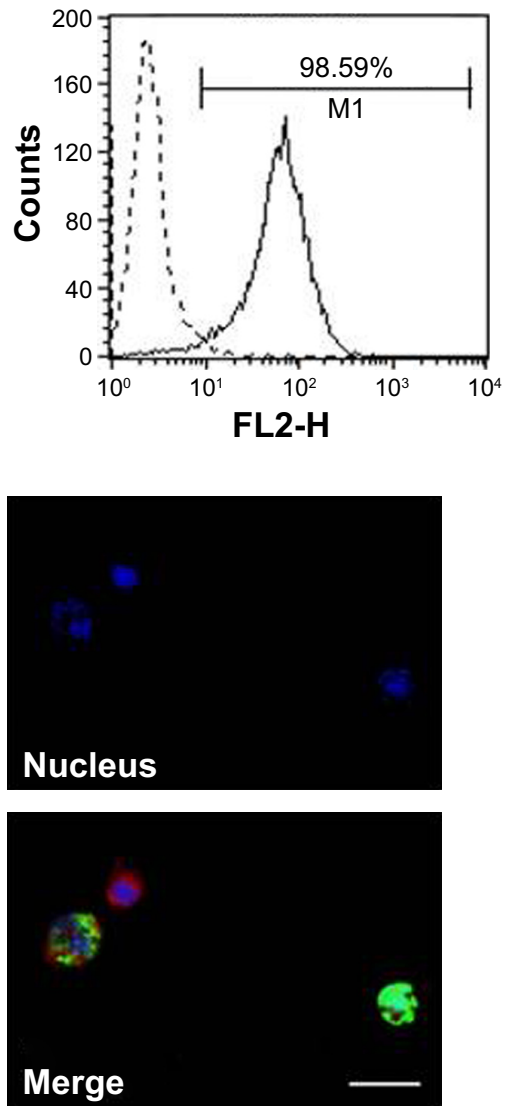

Figure I MECI cells' characterization and NPs' binding.

Notes: $(\mathbf{A})$ Cells $\left(5 \times 10^{5}\right)$ were incubated with PE-conjugated anti-CD5 or anti-CD79a and FITC-conjugated anti-CDI9 and anti-CD45 antibodies. The expression of CD20 on the surface of cells was confirmed using an anti-CD20 primary antibody (rituximab) and a FITC-conjugated antihuman secondary antibody. Samples were analyzed by FACSCalibur flow cytometer and data were analyzed by CELLQuest software. (B) FITC-conjugated NPs (green fluorescence) and cells labeled with Fast-Dil (red fluorescence) were incubated for I hour. DAPI was used to label nuclei (blue fluorescence). Samples were analyzed by cytometric analysis and with a Leica DM2000 optical microscope. Original magnification 630x.

Abbreviations: FITC, fluorescein isothiocyanate; FLI-H, green fluorescence, 530/30 nm bandpass filter; FL2-H, orange fluorescence, 585/42 nm bandpass filter; FSC-H, forward scatter height; MI, Marker I; NP, nanoparticle; PE, Phycoerythrin; SSC-H, side scatter height. 
and CD79a, by flow cytometry. The results showed that more than $90 \%$ of cells expressed CD19, CD20, CD45, and CD79a on their surface (Figure 1A). On the contrary, MEC1 cells did not express CD5, confirming the features of this cell line. ${ }^{26}$ To study NPs' binding specificity mediated by anti-CD20 antibody, FITC-conjugated anti-CD20 NPs were added to MEC1 cells for 1 hour and their binding was evaluated by cytometric analysis and fluorescence microscope. Under these conditions, targeted anti-CD20 NPs were able to target more than $90 \%$ of cells (Figure 1B). Targeted antiCD20 NPs binding to MEC1 cells were also confirmed by TEM analysis, which suggested NPs' internalization in a
A
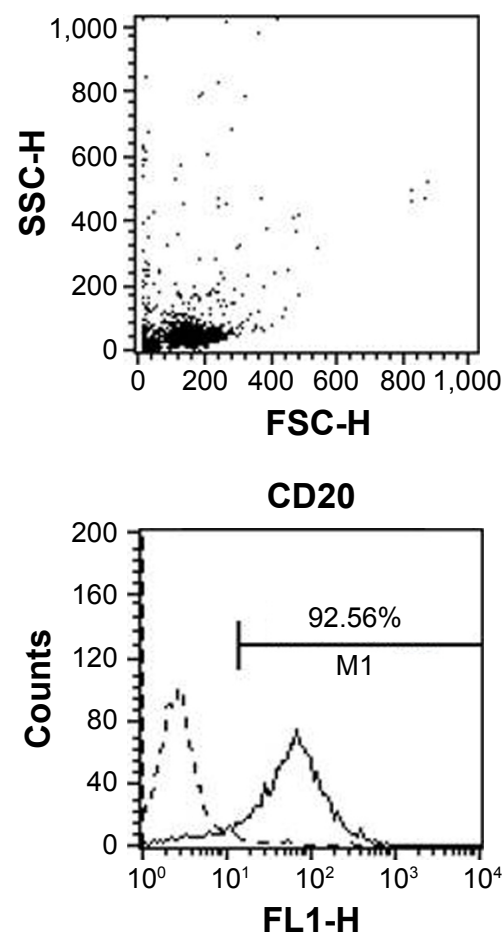

B

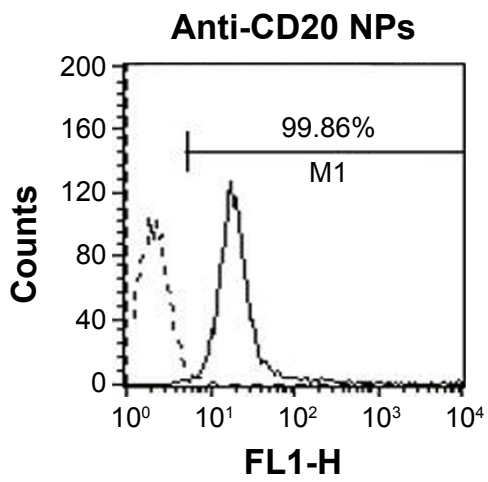

CD5

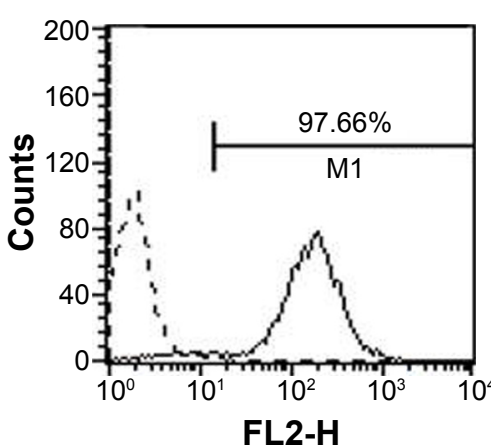

CD45

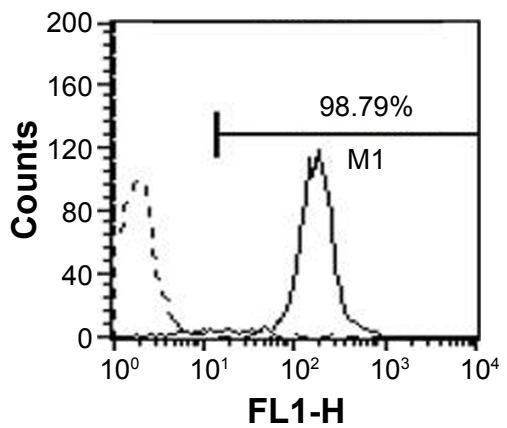

CD19

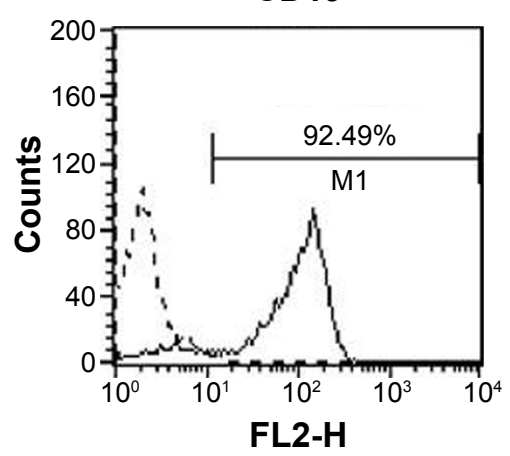

CD79a

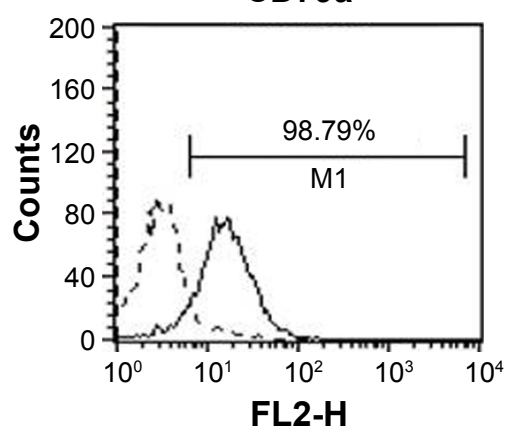

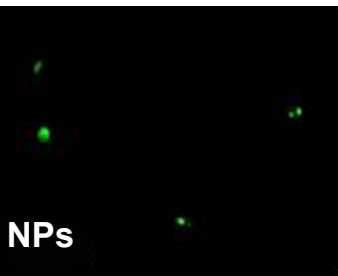
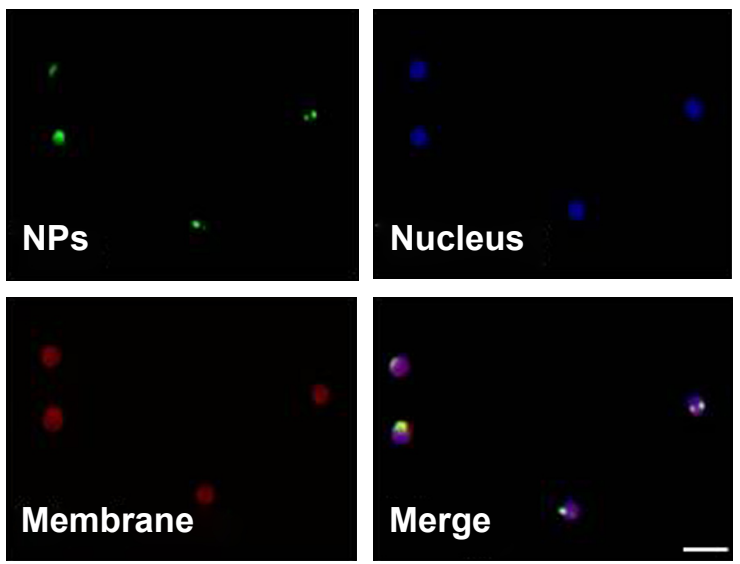

Figure 2 CLL patients' cell characterization and NP binding.

Notes: (A) Cells $5 \times 10^{5}$ were incubated with PE-conjugated anti-CD5 or anti-CD79a; and FITC-conjugated anti-CDI9 and anti-CD45 antibodies. The expression of CD20 on the surface of cells was confirmed using an anti-CD20 primary antibody and a FITC-conjugated antihuman secondary antibody. Samples were analyzed by FACSCalibur flow cytometer and data were analyzed by CELLQuest software. (B) FITC-conjugated NPs (green fluorescence) and cells labeled with Fast-Dil (red fluorescence) were incubated for I hour at $37^{\circ} \mathrm{C}$. NPs' binding was evaluated by cytometric analysis. DAPI was used to label nuclei (blue fluorescence) and samples were analyzed with a Leica DM2000 optical Microscope. Original magnification I00x.

Abbreviations: CLL, chronic lymphocytic leukemia; FITC, fluorescein isothiocyanate; FLI-H, green fluorescence, 530/30 nm bandpass filter; FL2-H, orange fluorescence, 585/42 nm bandpass filter; MI, Marker I; NP, nanoparticle; PE, Phycoerythrin; SSC-H, side scatter height. 
process different from endocytosis because of the absence of endocytotic vesicles. Moreover, NPs accumulated in the cells in only 1 hour, releasing their content in the cytoplasm but not in the nucleus, as already seen in lymphoma cells. ${ }^{17}$

\section{Characterization of CLL patients' cells and NPs' binding}

The expression of CD5, CD19, CD20, CD45, and CD79a on the surface of CLL-affected patients' cells was demonstrated by flow cytometry (Figure 2A). The results showed that more than $90 \%$ of cells express all the antigens on the cell surface, confirming CLL cells' immunophenotyping features. ${ }^{27} \mathrm{NPs}$ ' binding to leukemic cells was also evaluated by cytometric analysis: labeled with FITC, targeted anti-CD20 NPs were incubated with CLL cells for 1 hour at $37^{\circ} \mathrm{C}$ and the result showed NPs' ability to bind to more than $90 \%$ of patients' cells; these data were also confirmed by fluorescence microscope, evidencing the presence of targeted NPs in the cytoplasm of patients' B-cells (Figure 2B). Characterization of patient's cells is summarized in Supplementary material (Table S1).

\section{Development of a localized B-cell disorder mouse model}

To characterize NPs' biodistribution pattern, we set up a localized model of human B-cell disorder injecting $10^{7} \mathrm{MEC} 1$ cells sc in the flank of SCID mice. Tumor mass growth was measured thrice a week demonstrating its exponential growth (Figure 3A). Tumor mass was also analyzed by immunohistochemical analysis with anti-CD20, anti-CD5, anti-CD45, and anti-CD79a antibodies showing MEC1 cells' immunophenotyping features in vivo. In fact, MEC1-derived tumor mass was positive for the expression of CD20, CD45, and CD79a but the expression of CD5 was not detected (Figure 3B). In addition, immunohistochemical analysis on liver, spleen,

A
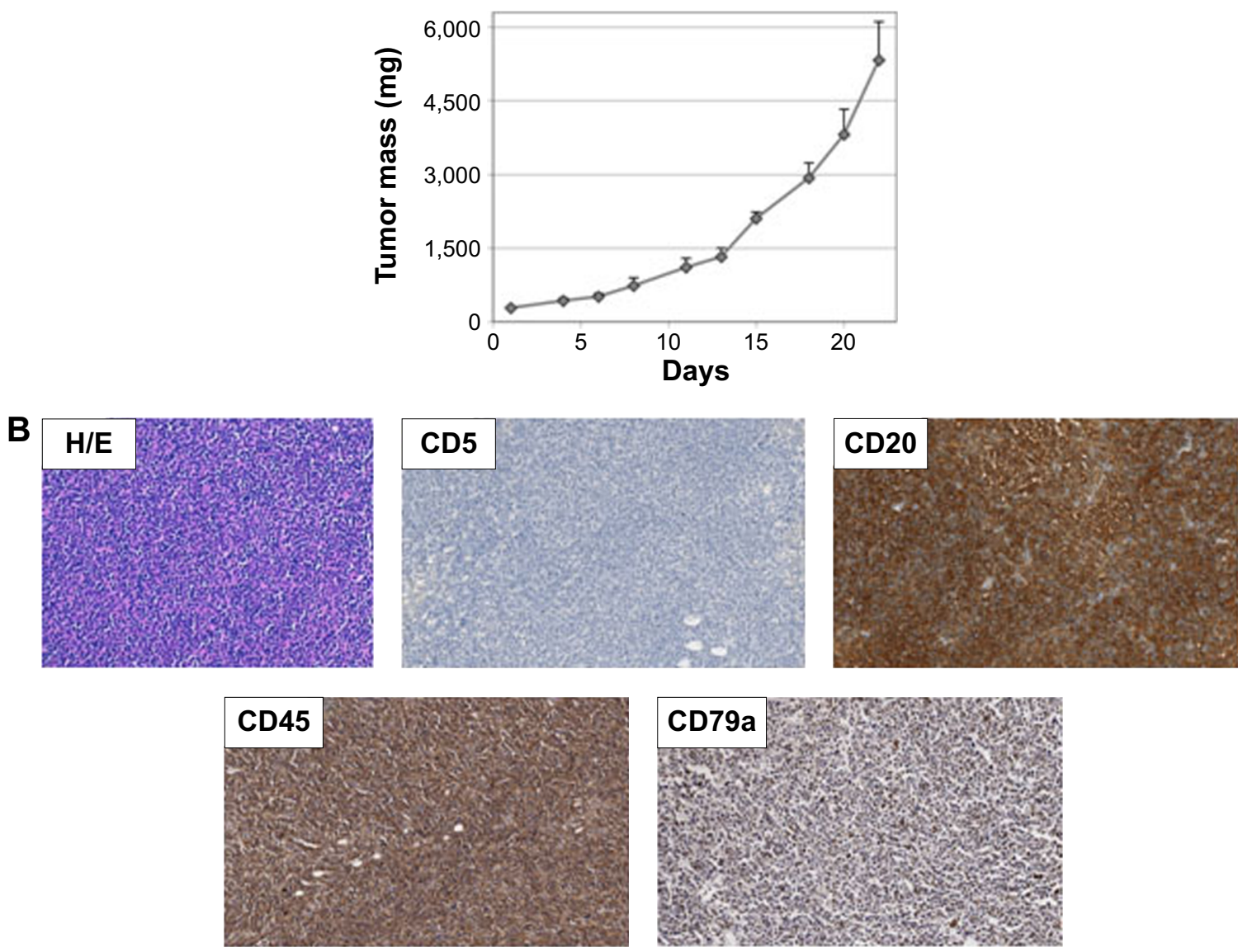

Figure 3 Characterization of a localized model of B-cell malignancies.

Notes: $(A)$ Mice $(n=6)$ were injected subcutaneously with $10^{7} \mathrm{MECl}$ cells and dimension of tumor mass was measured thrice per week. (B) Representative sections from subcutaneous masses stained with H/E, anti-CD5, anti-CD20, anti-CD45, and anti-CD79a monoclonal antibodies and 3,3'-diaminobenzidine chromogen. Original magnification 200x.

Abbreviation: $\mathrm{H} / \mathrm{E}$, hematoxylin and eosin. 


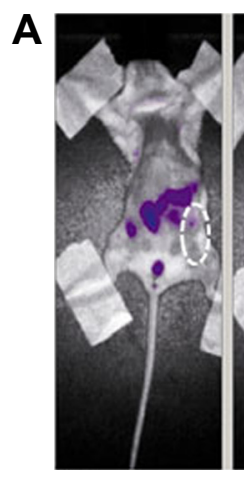

Pre-scan

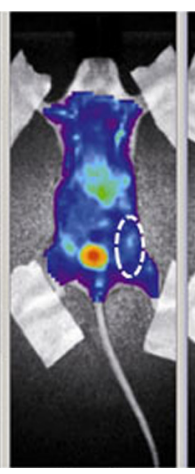

$10 \mathrm{~min}$

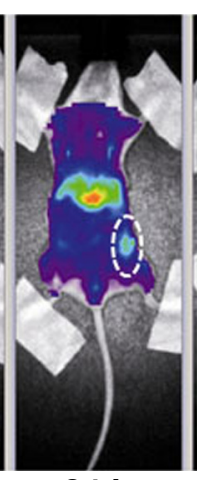

$24 \mathrm{~h}$

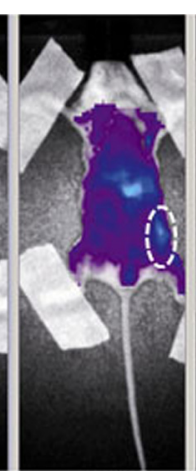

$48 \mathrm{~h}$

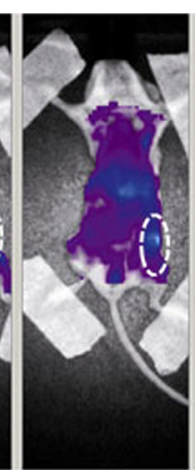

$96 \mathrm{~h}$

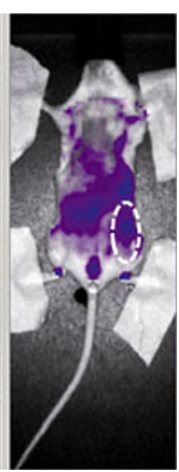

$168 \mathrm{~h}$
Intensity (NC)

$1.16 e+004$

$6.08 e+003$
B

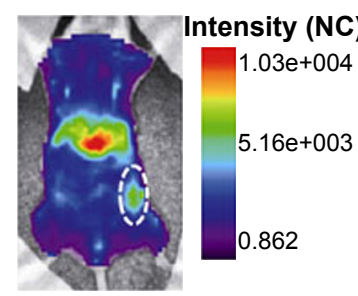

Anti-CD20 NPs

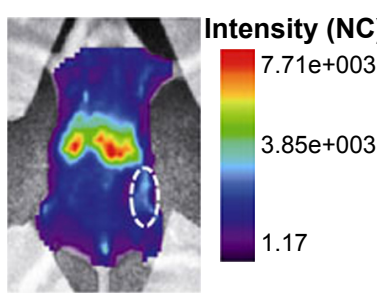

Untargeted NPs
C

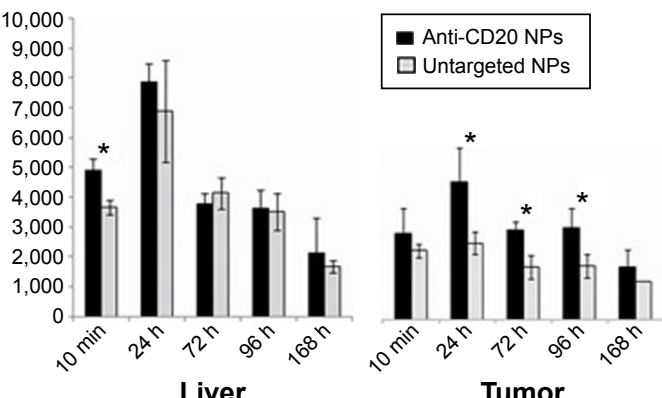

Figure 4 In vivo analysis.

Notes: (A) Biodistribution of Cy5.5-anti-CD20 NPs ( $0.3 \mathrm{nmol}$ Cy5.5, $125 \mathrm{mg}$ NPs) injected intravenously via the tail vein in a mouse model subcutaneously transplanted with the CLL cell line $\mathrm{MECI}$ (leukemic mouse model). Whole-body scan of a representative mouse in supine position is shown; fluorescence intensity images were acquired at the indicated time postinjection and are displayed in NC. The circles enclose the tumors. (B) Whole-body fluorescence intensity distribution in a representative leukemic mouse 24-hours postinjection of Cy5.5-anti-CD20 NPs and Cy5.5-untargeted NPs displayed in NC. The circles enclose the tumors. (C) The signal level profile describing the wash in/wash out of Cy5.5-anti-CD20 NPs and Cy5.5-untargeted NPs from liver and tumor is reported. Data are expressed as means \pm standard deviation of three independent experiments. $* P<0.05$ between Cy5.5-anti-CD20 NPs and Cy5.5-untargeted NPs.

Abbreviations: CLL, chronic lymphocytic leukemia; NC, normalized counts; NP, nanoparticle.

kidney, heart, brain, spinal cord, and bone marrow showed that MEC1 injection led to the formation of just a localized tumor mass without colonizing other tissues (data not shown).

\section{In vivo and ex vivo OI}

As shown in Figure 4A, a few minutes after intravenous injection of 1 nmol Cy5.5-anti-CD20 NPs, a diffuse distribution was observed throughout the body of the mouse. The contrast between tumor and surrounding tissue increased slowly, and was significantly higher than pre-contrast within the first 24 hours after injection and decreased over time. At 24 hours after injection, Cy5.5-anti-CD20 NPs allowed for clear delineation of the sc tumor tissue from the surrounding background tissue, leading to a signal-to-background ratio of approximately 7:1 (Figure 4B). Cy5.5-anti-CD20 NPs yield improved signal-to-background ratios (7:1) in comparison to Cy5.5-untargeted NPs (3:1). Interestingly, in vivo comparison of Cy5.5-untargeted NPs $(n=5)$ and Cy5.5-anti-CD20 NPs ( $n=5)$ showed a significant difference between the fluorescence intensity within the tumor site from
24 hours until 96 hours after injection (Figure 4C), while the intensity in the liver appeared to be almost the same (24 hours postinjection: difference of $\sim 13 \%, P=0.40$ ) and with no obvious overall difference in the NPs' uptake. The higher tumor targeting efficiency of Cy5.5-anti-CD20 NPs was further demonstrated by ex vivo analysis (Figure 5). The average fluorescence intensity over tumor tissues explanted at 168 hours after NPs administration showed a much higher levels of Cy5.5-anti-CD20 NPs than Cy5.5-untargeted NPs (difference 84\%, $P=0.0069$ ).

The selective localization of NPs within liver and tumor masses at the microscopic level was performed by confocal microscopy. The analyses of tissue cryosections isolated from mice sacrificed at 168 hours after administration are shown in Figure 5C. According to the significantly higher ex vivo-specific fluorescence signal, tumor masses of mice injected with Cy5.5-anti-CD20 NPs showed NIR fluorescent spots, consistent with clusters of NPs, while negligible NIR fluorescence was observed from the tumor mass sections of mice injected with Cy5.5-untargeted NPs. Fluorescence 
A

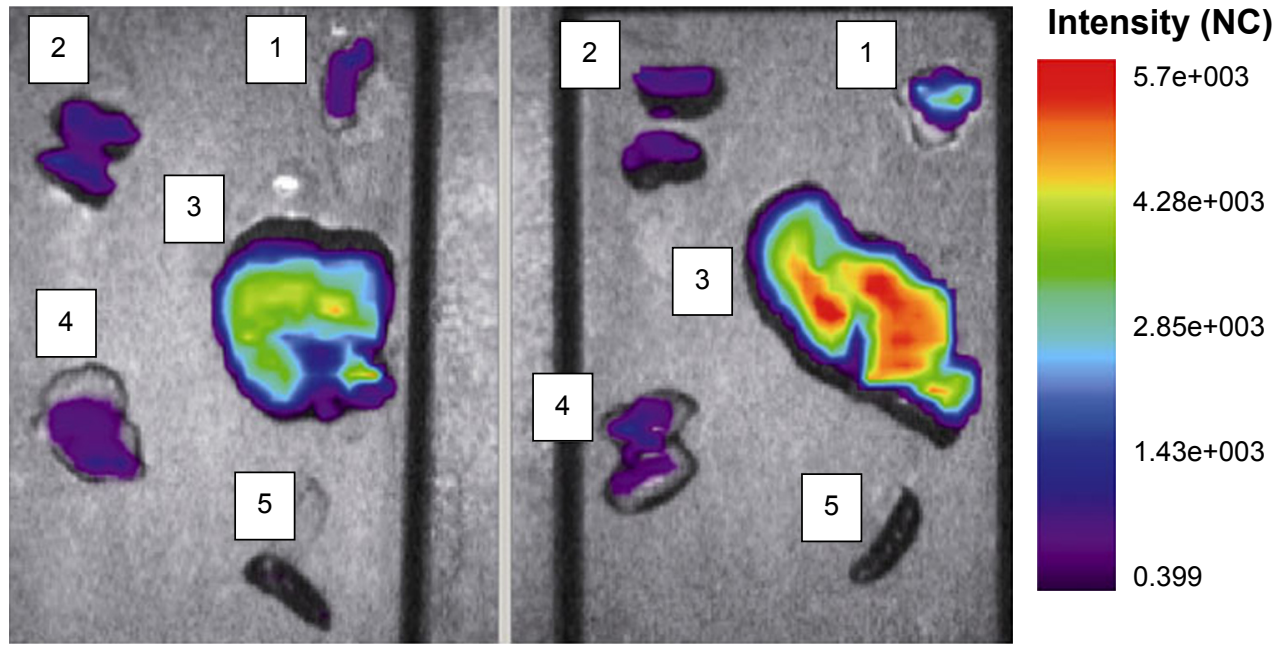

B

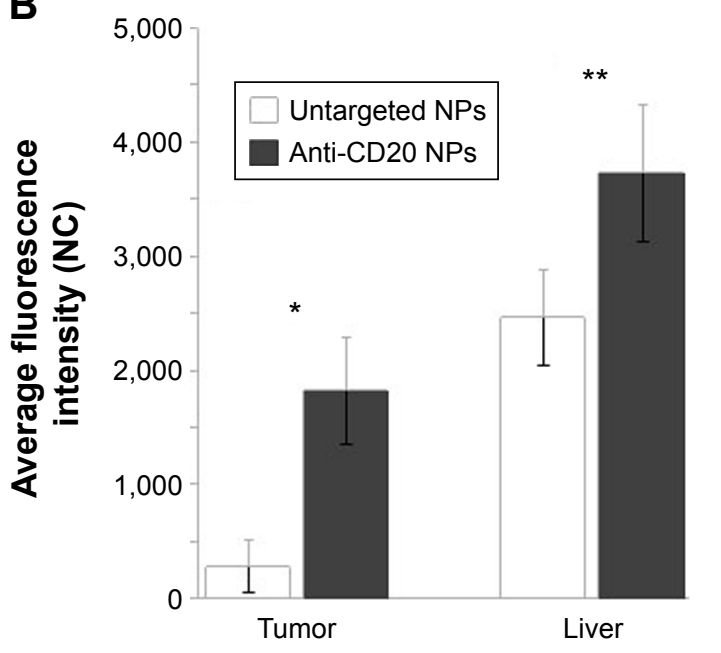

C

\section{Untargeted NPs Anti-CD20 NPs}

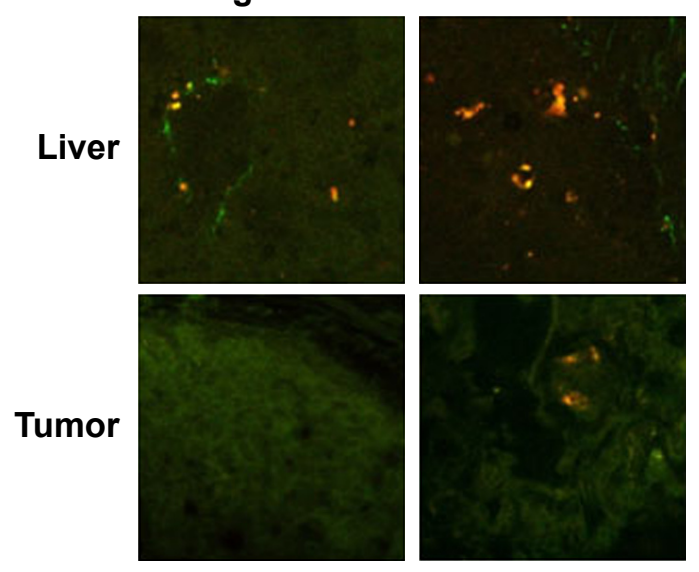

Figure 5 Ex vivo analysis.

Notes: (A) Ex vivo optical imaging of organs explanted at 168 hours after Cy5.5-untargeted NPs or Cy5.5-anti-CD20 NPs administration are shown - I: tumor mass; 2: kidney; 3: liver; 4: lung; 5: spleen. (B) Histogram representation of the average fluorescence intensity (NC) over tumor and liver tissues explanted at I68 hours after NP administration. $*$ Difference $84 \%, P$-value $=0.0069$; $*$ difference $34 \%, P$-value $=0.0056$. Data are expressed as means \pm standard deviation of three independent experiments. (C) Confocal microscopy images of explanted tumor mass' and liver's cryosections obtained from Cy5.5-untargeted NP and Cy5.5-anti-CD20 NP injections into mice. Tissue sections were also photographed through a green filter (FITC) to visualize background autofluorescence. Original magnification $600 \times$.

Abbreviations: FITC, fluorescein isothiocyanate; NC, normalized counts; NP, nanoparticle.

microscopy of liver sections revealed a non-homogeneously distributed NIR fluorescence signal, consistent with the presence of the NPs. All the microscopic observations were consistent with the in vivo and ex vivo fluorescence measurements. Ex vivo OI analysis of the explanted organs (ie, kidneys, heart, spleen, brain, lungs, stomach, intestine, liver) showed the preferential elimination of the NPs through the liver and a significant presence in the gastrointestinal tract (data not shown).

\section{Discussion}

We previously showed the impact of surface functionalization of biodegradable polymeric NPs on therapeutic efficacy in a
Burkitt's lymphoma model. ${ }^{17}$ Of note, hydroxychloroquine-/ chlorambucil-loaded NPs conjugated with an anti-CD20 antibody demonstrated a successful enhanced antitumor efficacy in vivo with respect to untargeted NPs, despite their comparable in vitro cytotoxic effect. In fact, the treatment of mice bearing B-cell lymphoma xenograft with anti-CD20 NPs selectively inhibited lymphoma growth due to the presence of the anti-CD20 targeting antibody. However, besides the enhanced therapeutic efficacy, there was no conclusive evidence supporting an increased tumor accumulation of targeted NPs.

In the present study, we were motivated to test whether this targeting strategy could provide in vivo tumor accumulation 
of a fluorescent contrast agent, potentially enabling tumor imaging. For this purpose, we first demonstrated in vitro the ability of targeted anti-CD20 NPs to bind to CLL cells. In detail, anti-CD20 NPs were able to bind high-CD20expressing cells such as MEC1 (mean fluorescence intensity: 285) as well as CLL-affected patients' cells which showed lower CD20 expression (mean fluorescence intensity: 77). Hence, we postulate that our anti-CD20 NPs could be very effective as a selective imaging probe for CD20-expressing tumors, and even serve as a personalized treatment strategy, by determining the expression level of these receptors in individual patients. As a proof of concept, we reported a specific internalization of anti-CD20 NPs by patients' CLL cells overexpressing CD20 receptor, thus confirming the importance of anti-CD20 antibody in the active targeting of NPs.

Then, we investigate the in vivo tumor-targeting properties of NP preparations in a localized murine model of B-cell malignancies. To establish a xenograft model, we focused on the biological characteristics of CLL cell line MEC1 cells and we tested their immunophenotypic profile by antigen evaluation, such as CD5, CD19, CD20, CD45, and CD79a, which are characteristics of MEC1 cells ${ }^{26}$ and are commonly used for the diagnosis of CLL. We investigated a model of sc injection of MEC1 in SCID mice. The results showed that a visible and well-localized tumor mass developed within a few weeks and recapitulated human tumor cell line culture in terms of cell morphology and immunohistochemical profile, offering a reasonable model for the study of local tumor uptake. However, these data are not in line with the finding that after the s.c. challenging of $\mathrm{MEC} 1$ in $\mathrm{Rag}^{-/-}$mice it was evident the formation of the tumor mass at the site of injection but also the development of a diffuse model of B-cell malignancy is evidenced. ${ }^{28}$ It is likely that the different backgrounds of the animals, and the presence of active immune cells like macrophage, polymorphonuclear leukocyte, and natural killer cells, prevented MEC1 distribution in other organs. ${ }^{29,30}$ Xenograft models were analyzed by TD OI after systemic Cy5.5-labeled NP administration. The results showed that the attachment of anti-CD20 tumor-targeting antibody on NPs significantly enhanced its total accumulation in tumors with respect to untargeted NPs. Region of interest analysis of the tumor site revealed intriguing differences in fluorescence contrast between untargeted- and anti-CD20 NPs. These findings indicate that the improved efficacy of anti-CD20 NPs most likely results from both interaction at the cellular level and enhanced tumor accumulation.

Of note, the incorporation of Cy5.5 as OI contrast agent within anti-CD20 NPs allowed us to clearly visualize the tumor mass within 24 hours after intravenous injection, which is critical for the successful development of imaging contrast nanoprobes. This provides evidence for the possible accumulation of these polymeric NPs to be used as a diagnostic probe in tumor microenvironment. These results also explain published and unpublished data about the efficacy of these polymeric NPs for drug delivery collected by our group. There are evidences about the need for a targeting agent on NPs' surface for an effective delivery of cytotoxic drugs and the cure of animal models of B-cell malignancies. Untargeted NPs were almost ineffective in the treatment different models of B-lymphoma and leukemia. This is probably not due to an enhance accumulation in the tumor microenvironment, but instead due to an increase in selective interaction between anti-CD20 NPs and tumor cell surface, and a possible increase in the loading of cytotoxic drug in tumor cell cytoplasm.

Anti-CD19 was also tested as an NP-targeting agent, ${ }^{31}$ alone or in combination with anti-CD20 antibodies. CD20 receptor was chosen as a molecular target because it is characterized by an high expression on cell surface of mature and tumoral B-cells. In addition, CD20 receptor is stable and provided with an high affinity for Rituximab. All these conditions suggested to focus on anti-CD20 NPs which result to be more efficient than untargeted NPs.

Furthermore, considering the capacity of these polymeric NPs to load not only chemotherapeutic drugs but also DNA vectors, miRNA, siRNA, and different probes, anti-CD20 NPs might become a highly attractive drug-delivery and imaging platform for future cancer theranostics.

\section{Conclusion}

In conclusion, NPs targeting the human B lymphocyte marker CD20 provide a strategy which could be extended as a generalized approach to improve the in vivo imaging of tumor mass, and to visualize the therapeutic agent delivery site in a wide variety of other CD20-expressing B-cell malignancies.

\section{Disclosure}

L Núñez is also affiliated to LNK Chemsolutions LLC. Biotarget and LNK Chemsolutions have commercial interests in the particle systems described in this work. The other authors report no conflicts of interest in this work.

\section{References}

1. Dawidczyk CM, Kim C, Park JH, et al. State-of-the-art in design rules for drug delivery platforms: lessons learned from FDA-approved nanomedicines. J Control Release. 2014;187:133-144.

2. Jain RK, Stylianopoulos T. Delivering nanomedicine to solid tumors Nat Rev Clin Oncol. 2010;7:653-664. 
3. Bartlett DW, Su H, Hildebrandt IJ, Weber WA, Davis ME. Impact of tumor-specific targeting on the biodistribution and efficacy of siRNA nanoparticles measured by multimodality in vivo imaging. Proc Natl Acad Sci U S A. 2007;104:15549-15554.

4. Chattopadhyay N, Fonge H, Cai Z, et al. Role of antibody-mediated tumor targeting and route of administration in nanoparticle tumor accumulation in vivo. Mol Pharm. 2012;9(8):2168-2179.

5. Sanna V, Pala N, Sechi M. Targeted therapy using nanotechnology: focus on cancer. Int J Nanomedicine. 2014;9:467-483.

6. Ruoslahti E, Bhatia SN, Sailor MJ. Targeting of drugs and nanoparticles to tumors. J Cell Biol. 2010;188:759-768.

7. Kirpotin DB, Drummond DC, Shao Y, et al. Antibody targeting of long-circulating lipidic nanoparticles does not increase tumor localization but does increase internalization in animal models. Cancer Res. 2006;66:6732-6740.

8. Matsumura Y, Maeda H. A new concept for macromolecular therapeutics in cancer chemotherapy: Mechanism of tumoritropic accumulation of proteins and the antitumor agent smancs. Cancer Res. 1986;46:6387-6392.

9. Gu G, Xia H, Hu Q, et al. PEG-co-PCL nanoparticles modified with MMP-2/9 activatable low molecular weight protamine for enhanced targeted glioblastoma therapy. Biomaterials. 2013;34:196-208.

10. Gao H, Yang Z, Zhang S, et al. Ligand modified nanoparticles increases cell uptake, alters endocytosis and elevates glioma distribution and internalization. Sci Rep. 2013;3:2534.

11. Valencia PM, Pridgen EM, Rhee M, Langer R, Farokhzad OC, Karnik R. Microfluidic platform for combinatorial synthesis and optimization of targeted nanoparticles for cancer therapy. ACS Nano. 2013;7:10671-10680.

12. Pirollo KF, Chang EH. Does a targeting ligand influence nanoparticle tumor localization or uptake? Trends Biotechnol. 2008;26:552-558.

13. Theek B, Gremse F, Kunjachan S, et al. Characterizing EPR-mediated passive drug targeting using contrast-enhanced functional ultrasound imaging. J Control Release. 2014;182:83-89.

14. Kunjachan S, Pola R, Gremse F, et al. Passive versus active tumor targeting using RGD- and NGR-modified polymeric nanomedicines. Nano Lett. 2014;14:972-981.

15. Wang AZ, Langer R, Farokhzad OC. Nanoparticle delivery of cancer drugs. Anпu Rev Med. 2012;63:185-198.

16. Cai W, Gao T, Hong H, Sun J. Applications of gold nanoparticles in cancer nanotechnology. Nanotechnol Sci Appl. 2008;1:17-32.

17. Mezzaroba N, Zorzet S, Secco E, et al. New potential therapeutic approach for the treatment of B-Cell malignancies using chlorambucil/ hydroxychloroquine-loaded anti-CD20 nanoparticles. PLoS One. 2013; 8(9):e74216.

18. Marín GH, Mansilla E, Mezzaroba N, et al. Exploratory study on the effects of biodegradable nanoparticles with drugs on malignant $\mathrm{B}$ cells and on a human/mouse model of Burkitt lymphoma. Curr Clin Pharmacol. 2010;5:246-250.
19. Rampazzo E, Boschi F, Bonacchi S, et al. Multicolor core/shell silica nanoparticles for in vivo and ex vivo imaging. Nanoscale. 2012;4: 824-830.

20. Macor P, Secco E, Mezzaroba N, et al. Bispecific antibodies targeting tumor-associated antigens and neutralizing complement regulators increase the efficacy of antibody-based immunotherapy in mice. Leukemia. 2015;29(2):406-414.

21. Zabucchi G, Soranzo MR, Menegazzi R, et al. Eosinophil peroxidase deficiency: morphological and immunocytochemical studies of the eosinophil-specific granules. Blood. 1992;80:2903-2910.

22. Biffi S, Dal Monego S, Dullin C, et al. Dendritic polyglycerolsulfate near infrared fluorescent (NIRF) dye conjugate for non-invasively monitoring of inflammation in an allergic asthma mouse model. PLoS One. 2013;8:e57150.

23. Biffi S, Petrizza L, Rampazzo E, et al. Multiple dye-doped NIR-emitting silica nanoparticles for both flow cytometry and in vivo imaging. RSC Adv. 2014;4:18278-18285.

24. Biffi S, Garrovo C, Macor P, et al. In vivo biodistribution and lifetime analysis of cy5.5-conjugated rituximab in mice bearing lymphoid tumor xenograft using time-domain near-infrared optical imaging. Mol Imaging. 2008;7:272-282.

25. Florena AM, Tripodo C, Iannitto E, Porcasi R, Ingrao S, Franco V. Value of bone marrow biopsy in the diagnosis of essential thrombocythemia. Haematologica. 2004;89:911-919.

26. Stacchini A, Aragno M, Vallario A, et al. MEC1 and MEC2: two new cell lines derived from B-chronic lymphocytic leukaemia in prolymphocytoid transformation. Leuk Res. 1999;23:127-136.

27. Hallek M, Cheson BD, Catovsky D, et al. Guidelines for the diagnosis and treatment of chronic lymphocytic leukemia: a report from the International Workshop on Chronic Lymphocytic Leukemia updating the National Cancer Institute-Working Group 1996 guidelines. Blood. 2008;111:5446-5456.

28. Bertilaccio MT, Scielzo C, Simonetti G, et al. A novel Rag2-/-gammac-/-xenograft model of human CLL. Blood. 2010;115:1605-1609.

29. Mombaerts P, Iacomini J, Johnson RS, Herrup K, Tonegawa S, Papaioannou VE. RAG-1-deficient mice have no mature B and T lymphocytes. Cell. 1992;68:869-877.

30. Bancroft GJ, Kelly JP. Macrophage activation and innate resistance to infection in SCID mice. Immunobiology. 1994;191:424-431.

31. Mansilla E, Marin GH, Nuñez L, et al. The lysosomotropic agent, hydroxychloroquine, delivered in a biodegradable nanoparticle system, overcomes drug resistance of B-chronic lymphocytic leukemia cells in vitro. Cancer Biother Radiopharm. 2010;25:97-103.

32. Bray SJ. Notch signalling: a simple pathway becomes complex. Nat Rev Mol Cell Biol. 2006;7(9):678-689.

33. Gattei V, Bulian P, Del Principe MI, et al. Relevance of CD49d protein expression as overall survival and progressive disease prognosticator in chronic lymphocytic leukemia. Blood. 2008;111(2):865-873. 


\section{Supplementary material}

Table SI Characterization of patient's cells

\begin{tabular}{|c|c|c|c|c|c|c|c|c|c|c|c|c|c|}
\hline Code & CDI9 & CD5 & CD49d & CD20 & CD38 & ZAP-70 & TP53 M & SF3B I $M$ & MYD88 M & $\begin{array}{l}\text { NOTCH } \\
\text { status }\end{array}$ & $\begin{array}{l}\text { IGHV } \\
\text { status }\end{array}$ & $\begin{array}{l}\text { FISH } \\
\text { status }\end{array}$ & RB I \\
\hline $\mathrm{Pt} \mathrm{I}$ & 89.4 & 99.5 & 99.1 & 96 & 99.2 & & 0 & 0 & & & na & TRII2 & \\
\hline Pt 2 & 90 & 99.9 & 1.3 & 99.4 & 0.5 & 12.5 & 0 & 0 & & 0 & UM & DELII & \\
\hline Pt 3 & 89.6 & 99.5 & 0.8 & 91.6 & 8 & 3.2 & 0 & 0 & & 0 & UM & DELI3, DELII & \\
\hline $\mathrm{Pt} 4$ & 55.6 & 83.7 & 39 & 99.3 & 40.3 & 12 & 0 & 0 & & & na & DELI3 & 0 \\
\hline Pt 5 & 60.8 & 70.6 & 35.3 & 98.4 & 45.8 & 10 & 0 & 0 & & & na & NORM & \\
\hline Pt 6 & 99 & 64.8 & 2.2 & 98.3 & 12.2 & 8.7 & 0 & 0 & & 0 & M & DELI3, DELI7 & \\
\hline Pt 7 & 94.7 & 97.4 & 32.3 & 99.2 & 15 & I & 0 & 0 & & & $M$ & DELI3 omo & \\
\hline Pt 8 & 64.8 & 91.2 & 97 & 92.3 & 26 & 10 & 0 & 0 & 0 & 0 & $M$ & DELI 3 & 0 \\
\hline
\end{tabular}

Notes: CD19, CD5, CD49d, CD20, CD38 expressions are reported as percentage of positive cells. NOTCH status was determined by ARMS-PCR, conventional, and nextgeneration sequencing. IGHV status was established according to conventional cut-off; $\mu \mathrm{m}$, IGHV unmutated, m, IGHV mutated. FISH status was determined according to Bray. ${ }^{32}$ Other parameter expression was determined as reported in Gattei et al. ${ }^{33}$

Abbreviations: ARMS-PCR, Amplification Refractory Mutation System-Polymerase Chain Reaction; FISH, Fluorescence in situ hybridization; IGHV, Immunoglobulin heavy chain variable region genes; na, not available; $M$, mutated; UM, unmutated.

\section{Publish your work in this journal}

The International Journal of Nanomedicine is an international, peerreviewed journal focusing on the application of nanotechnology in diagnostics, therapeutics, and drug delivery systems throughou the biomedical field. This journal is indexed on PubMed Central, MedLine, CAS, SciSearch $®$, Current Contents $\AA /$ Clinical Medicine,
Journal Citation Reports/Science Edition, EMBase, Scopus and the Elsevier Bibliographic databases. The manuscript management system is completely online and includes a very quick and fair peer-review system, which is all easy to use. Visit http://www.dovepress.com/ testimonials.php to read real quotes from published authors.

\footnotetext{
Submit your manuscript here: http://www.dovepress.com/international-journal-of-nanomedicine-journal
} 